\title{
Determining Bone Bruises of the Knee with Magnetic Resonance Imaging
}

\author{
Bekir Yavuz Uçar ${ }^{*}, 1$, Serdar Necmioğlu ${ }^{1}$, Mehmet Bulut ${ }^{1}$, İbrahim Azboy ${ }^{1}$, Abdullah Demirtaş ${ }^{1}$ and \\ Hatice Gümüş²
}

\author{
${ }^{\prime}$ Department of Orthopaedic \& Traumatology, Medical Faculty, Dicle University, Turkey \\ ${ }^{2}$ Department of Radiology, Medical Faculty, Dicle University, Turkey
}

\begin{abstract}
Introduction: Injuries that develop secondary to minor traumas and cannot be detected via direct examination methods, but are detected via advanced imaging methods, such as magnetic resonance (MR) imaging, are called occult bone injuries or bone bruises. In such injuries, diagnostic arthroscopy usually does not reveal any pathology. MR imaging methods are quite beneficial for the diagnosis of such clinical conditions, which cause acute pain and restriction of motion. The present study aimed to assess occult bone injuries via MR imaging in patients who presented with minor knee trauma.
\end{abstract}

Patients and Methods: Twelve patients who presented with minor knee trauma were included in the study. Etiological factors in these patients included walking a long distance, falls, and minor trauma. All patients underwent physical examinations, direct radiological imaging, MR imaging, and diagnostic arthroscopy.

Results: Direct radiographs of the patients showed no pathological fracture. Bone marrow changes detected on the MR images were classified according to Lynch's classification as Type I lesions in nine patients and Type II lesions in three patients.

Conclusion: We suggest that MR imaging methods should be the gold standard for the diagnosis of minor traumatic bruise injuries of the knee.

Keywords: Knee, trauma, magnetic resonance imaging, bone bruise.

\section{INTRODUCTION}

Lesions that are covered by cartilage or other formations, and thus, cannot be visualised on traditional radiographs or via direct examination methods, such as arthroscopy, are referred to as occult bone injuries, occult interosseous fractures, or bone bruises.

Since the late $1980 \mathrm{~s}$, occult bone injuries have been diagnosed in association with soft tissue injuries of the knee, particularly with the rupture of the anterior cruciate ligament, and less commonly, with injuries of the hip joint and foot. Such occult bone injuries, also called bone bruise, present with bleeding, oedema, and infarct in the subchondral bone marrow due to micro-trabecular fractures [1]. Diagnosis of such lesions has become more common, particularly with the use of magnetic resonance (MR) imaging methods [2-5]. A typical occult bone injury is observed as a signal loss on T1 images and increased signal content on T2 images, due to the increased water content in the damaged bone marrow. Bone bruise and bone marrow oedema could be distinguished primarily based on trauma history, as their radiological features are almost indistinguishable. The sensitivity and specificity of MR imaging methods in detecting bone bruises

*Address correspondence to this author at the Department of Orthopaedic \& Traumatology, Medical Faculty, Dicle University, 21280, Sur, Diyarbakir, Turkey; Tel: 00904122488001; Fax: 00904122488111;

E-mail: drbyucar@yahoo.com of the knee have been reported to be $83 \%$ - $96 \%$ and $86 \%$ - $96 \%$, respectively [6].

Bone bruises of the knee may cause substantial clinical entities, as they cause acute pain and functional loss in the joint $[7,8]$. In terms of the natural history of bone bruises, it has been reported that such lesions resolve within a period ranging from 6-12 weeks [9] to 12-14 weeks [10], and even to 24 months [11]. Despite the lack of a definite consensus on the follow-up periods of such lesions via MR imaging methods, the clinical reflections and importance of longterm, non-healing lesions are not known. The present study aimed to assess occult bone injuries using MR imaging in patients presenting with minor knee trauma.

\section{MATERIALS AND METHODS}

\section{Study Design}

We retrospectively reviewed cases of bone bruises of the knee based on MR imaging performed following minor traumatic injury. Twelve patients (3 females, 9 males), between January 2010 and December 2010 were enrolled in this study. The patients presented to our clinic with persistent complaints despite conservative therapy. Exclusion criteria were as follows: any previous symptom or pathology of the knee, severe injury, rheumatoid arthritis that caused degenerative arthropathy or premature degenerative arthritis, sickle cell, psoriasis, and haemophilia. Patients with 
pathology of the menisci or ligaments were also excluded from the study.

In clinical evaluations, range of joint motion (ROM) and knee circumference were measured in all patients to evaluate arthropathy or hypertrophy. In addition, McMurray's, Apley's, and Steinman's tests were used to examine the meniscus; varus and valgus stress tests were used to examine the collateral ligament; and Lachman's, Slocum's, and pivot shift tests were used to examine the anterior cruciate ligament. Radiographs of all patients were evaluated, and mechanical axes were measured. Finally, the patients underwent MR imaging. The obtained MR images were classified according to the criteria defined by Lynch et al., [12]. On short echo time (TE) images, Type I lesions showed a signal intensity loss that was primarily located within the medullar cavity of the bone, without cortical interruption. A Type II lesion was defined as a loss of signal intensity associated with interruptions in the black cortical line; such an abnormality was defined in the present study as a fracture combined with bone bruise. On short TE images, a type III lesion was defined as a loss of signal intensity without definite cortical interruption in the bone regions adjacent to the cortex.

Although simple analgesics were the first treatment choice for these patients, different drugs were also used if needed. Intra-articular injections, steroids, and viscosupplementation were not used. All of the patients received standard physiotherapy, and they were asked not to participate in regular sports, but to continue their normal activities as long as the pain permitted. Diagnostic arthroscopy was performed a mean of 18 days (range, 15-21 days) after the initial presentation in five patients in whom improvement was not achieved with conservative therapy. MR imaging was repeated 16-18 weeks after the first presentation in all patients.

All the patients gave informed consent prior to being included in the study. The study was authorized by the local ethical committee and was performed in accordance with the ethical standards of the 1964 Declaration of Helsinki, as revised in 2000 .

\section{Magnetic Resonance Imaging Technique}

Standard knee MR imaging included coronal T1 sequences, sagittal T2 sequences, and sagittal-coronal T2 fatsuppressed sequences. The diagnosis of occult bone bruise was established based on the presence of decreased signal intensity on T1-weighted sequences and increased signal intensity on T2-weighted sequences and fat-suppressed T2weighted sequences.

\section{RESULTS}

In the present study, the data of nine male and three female patients with a mean age of 32 years (range, 24-51 years) were reviewed. All of the patients were admitted with minor traumatic knee injury. The etiological factors of the injuries before the patients complained of knee pain were walking a long distance in six patients, falling from a 1meter height in three patients, and minor knee trauma in three patients. Moreover, one of the patients had a history of surgery for rupture of the anterior cruciate ligament eight years prior; the other patients had no history of surgery on the knee or other extremities.

The mean restriction of ROM in patients was $15^{\circ}$ (range, $\left.10-40^{\circ}\right)$. Minimal joint effusion was observed in three patients. Joint puncture performed on these patients revealed no microbiological or histological pathology. No pathological signs were detected on the clinical and radiological evaluations of the patients (Fig. 1). The mechanical axis was within the normal range in all patients.

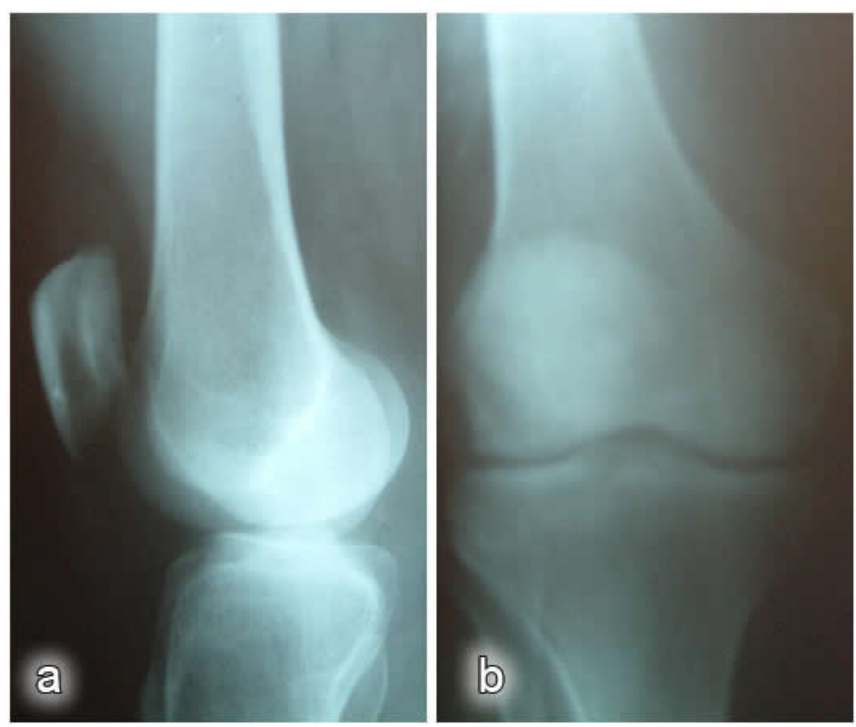

Fig. (1). Post-traumatic direct radiographs: a) lateral view; b) anteroposterior view.

Bone marrow changes detected on the MR images were classified as Type I lesions in nine patients and Type II lesions in three patients (Figs. 2, 3).

Diagnostic arthroscopy was performed in five patients in whom improvement was not achieved with conservative therapy. In these patients, the lesions were classified as Type I according to the International Cartilage Repair Society (ICRS) classification [13]. All patients treated conservatively. The lesions improved in all patients within a mean period of six months (range, 4-12 months) after the trauma.

\section{DISCUSSION}

Currently, there is no definite consensus on the natural history of bone injuries secondary to minor knee traumas. Injuries that occur after a low-energy trauma generally resolve in a short time [9], and in such patients, precautions that prevent weight load on the knee joint are sufficient for the treatment. Improvements observed in our patients within a mean period of six months after the trauma were consistent with previous reports.

Radiographs, which are beneficial in detecting bone fractures following traumatic knee injuries, are inadequate, particularly in detecting soft tissue pathologies. On the other hand, MR imaging, a non-invasive method that shows knee joint injuries quite accurately, has begun to be used widely in the last few decades. 

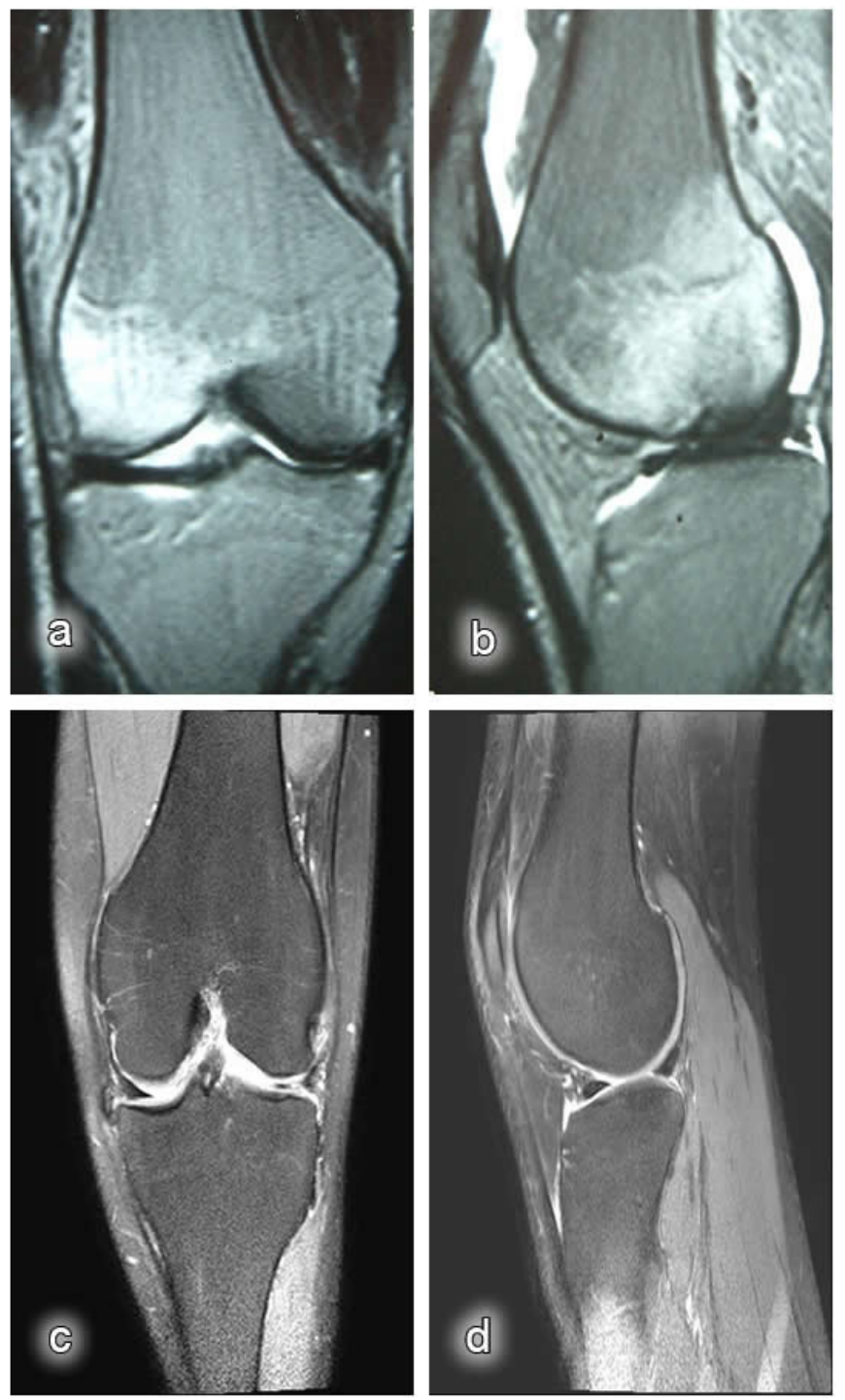

Fig. (2). Magnetic resonance images: a) post-traumatic coronal MR image of the knee (bone bruise in the lateral condyle); b) posttraumatic sagittal MR image of the knee (bone bruise in the lateral condyle); c) coronal MR image of the knee obtained at the last control visit (after nine months); d) sagittal MR image of the knee obtained at the last control visit (after nine months).

No remarkable pathology was noted on the radiographs obtained after minor knee trauma in our patients. MR images revealed bone contusions characterised by low signal intensity on T1-weighted images and high signal intensity on T2-weighted images, although a remarkable fracture line was not detected on the bony parts exposed to minor trauma. Contusions that could not be detected on the direct radiographs were the most common lesions detected on the MR images.

Based on the available data on biomechanics of the knee joint, it is known that intensive mechanical stress could cause an overload on the subchondral region and lead to contusion-type injuries in the event of weakness of the muscle mass protecting the joint structure. This might explain the injuries that occurred due to walking for a longer time than normal in six patients in our series. Bruise injuries due to minor traumas in the other patients were confirmed by MR imaging findings.
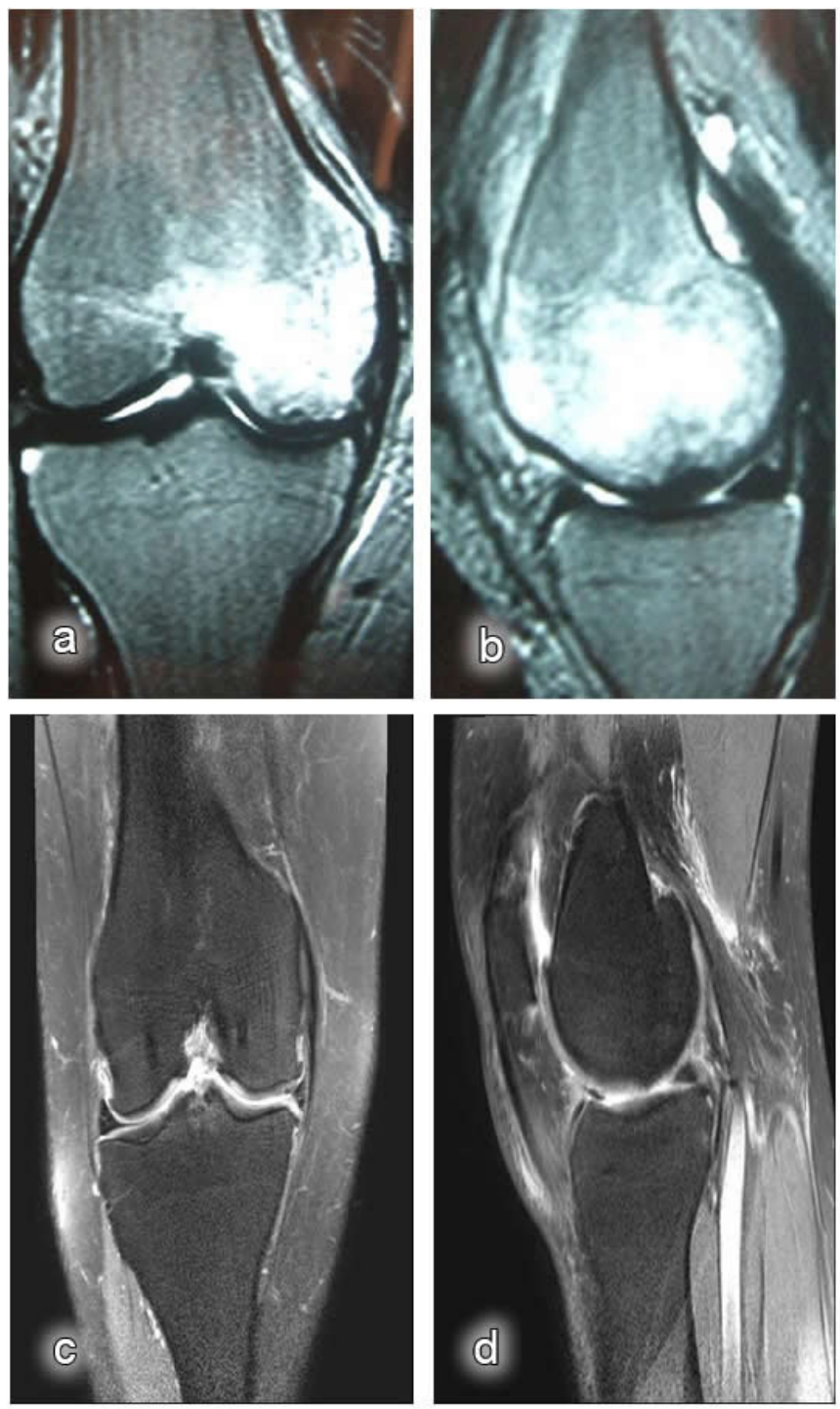

Fig. (3). Magnetic resonance images: a) post-traumatic coronal MR image of the knee (bone bruise in the medial condyle); b) posttraumatic sagittal MR image of the knee (bone bruise in the medial condyle); c) coronal MR image of the knee obtained at the last control visit (after 12 months); d) sagittal MR image of the knee obtained at the last control visit (after 12 months).

In their study, Maurer et al., [14] found that MR imaging in acute knee trauma improved the diagnostic certainty of clinicians and reduced the number of diagnostic arthroscopies. This finding was also confirmed by BuiMansfield et al., [15], and it was reported that MR imaging performed before arthroscopy of the knee reduced costs. In the present study, positive results were obtained via MR images of the patients in whom radiographs showed no pathological findings, and these patients were classified as Type I, based on the arthroscopic findings [16]. Therefore, it can be assumed that using MR imaging in acute minor knee traumas might prevent unnecessary diagnostic arthroscopy.

\section{CONFLICT OF INTEREST}

The author confirms that this article content has no conflict of interest. 


\section{ACKNOWLEDGEMENTS}

Declared none.

\section{REFERENCES}

[1] Rangger C, Kathrein A, Freund MC, et al. Bone bruise of the knee: histology and cryosections in 5 cases. Acta Orthop Scand 1998; 69: 291-4.

[2] Berger PE, Ofstein RA, Jackson DW, et al. MRI demonstration of radiographically occult fractures: what have we been missing? Radio Graphics 1989; 9: 407-36.

[3] Yao L, Lee JK. Occult intraosseous fracture:detection with MRI. Radiology 1988; 167: 749-51.

[4] Mink JH, Deutsch AL. Occult cartilage and bone injuries of the knee: Detection, classification and assessment with MR imaging. Radiology 1989; 170: 823-9.

[5] Vellet AD, Marks PH, Fowler PJ, et al. Occult post-traumatic osteochondral lesions: Prevalence, classification and short-term sequelae evaluated with MR imaging. Radiology 1991; 178: 271-6.

[6] Arndt WF, Truax AL, Barnett FM, et al. Diagnosis of bone contusions of the knee: comparison of coronal T2-weighted fast spin-echo with fat saturation and fast spin-echo STIR images with conventional STIR images. AJR Am J Roentgenol 1996; 166(1): 119-24.

[7] Vincken PW, Ter Braak BP, van Erkel AR, et al. Clinical consequences of bone bruise around the knee. Eur Radiol 2006; 16(1): 97-107.

[8] Aigner N, Meizer R, Meraner D, et al. Tapping test in patients with painful bone marrow edema of the knee. Clin J Pain 2008; 24(2): $131-4$
[9] Miller MD, Osborne JR, Gordon WT, et al. The natural history of bone bruises. A prospective study of magnetic resonance imagingdetected trabecular microfractures in patients with isolated medial collateral ligament injuries. Am J Sports Med 1998; 26: 15-9.

[10] Davies NH, Niall D, King LJ, et al. Magnetic resonance imaging of bone bruising in the acutely injured knee-short-term outcome. Clin Radiol 2004; 59: 439-45.

[11] Roemer FW, Bohndorf K. Long-term osseous sequelae after acute trauma of the knee joint evaluated by MRI. Skeletal Radiol 2002; 31: 615-23.

[12] Lynch TCP, Crues JV, Morgan FW, et al. Bone abnormalities of the knee: prevalence and significance at MR imaging. Radiology 1989; 171: 761-6.

[13] Cameron ML, Briggs KK, Steadmann JR. Reproducibility and reliability of the outerbridge classification for grading chondral lesions of the knee arthroscopically. Am J Sports Med 2003; 31(1): 83-6.

[14] Wright RW, Phaneuf MA, Limbird TJ, et al. Clinical outcome of isolated subcortical trabecular fractures (bone bruise) detected on magnetic resonance imaging in knees. Am J Sports Med 2000; 28 : 663-7.

[15] Maurer EJ, Kaplan PA, Dussault RG, et al. Acutely injured knee: effect of MR imaging on diagnostic and therapeutic decisions. Radiology 1997; 204: 799-805.

[16] Bui-Mansfield LT, Youngberg RA, Warme W, et al. Potential cost savings of MR imaging obtained before arthroscopy of the knee: evaluation of 50 consecutive patients. AJR Am J Roentgenol 1997; 168: 913-8.

(C) Uçar et al.; Licensee Bentham Open.

This is an open access article licensed under the terms of the Creative Commons Attribution Non-Commercial License (http://creativecommons.org/licenses/by-nc/3.0/) which permits unrestricted, non-commercial use, distribution and reproduction in any medium, provided the work is properly cited. 